\title{
Separação Entre Propriedade e Decisão de Gestão nas Cooperativas Agropecuárias Brasileiras
}

\author{
Davi Rogério de Moura Costa ${ }^{1}$, Fabio Ribas Chaddad $^{2}$ e \\ Paulo Furquim de Azevedo ${ }^{3}$
}

\begin{abstract}
Resumo: A visão predominante na teoria econômica é que organizações de propriedade difusa e complexas apresentam melhor desempenho se forem separados os direitos ao lucro residual das decisões de gestão. Nos países de economia desenvolvida, os modelos de governança corporativa das cooperativas agropecuárias de propriedade difusa e complexas, tal como já informado pela literatura econômica, promovem a desvinculação dos cooperados da gestão da empresa. Em contraposição, no Brasil, embora não haja estudos sistemáticos sobre o tema, evidências pontuais indicam que essas organizações concentram a propriedade e decisões de gestão. A possível divergência entre os modelos de governança utilizados nos diferentes países revela a necessidade de mensuração do grau de separação entre propriedade e decisão de gestão em cooperativas agropecuárias brasileiras, tarefa a que se dedica este artigo. A partir de dados coletados junto a 77 cooperativas agropecuárias, identificou-se que diferentes modelos de governança coexistem no Brasil. Embora grande parcela de cooperativas tenha governança concentrada, há um grupo que já adota modelos de governança que promovem a separação parcial de jure ou de facto entre propriedade e decisão de gestão. Esses resultados revelam a necessidade de pesquisas futuras voltadas a identificar os determinantes da variabilidade de modelos de governança nas cooperativas brasileiras.
\end{abstract}

Palavras-chaves: cooperativismo, controle e governança corporativa.

Abstract: Contract and Organizational theories establish that complex organizations with diffuse ownership, such as agriculture cooperatives, should benefit from the separation of ownership and decision control. In developed economies, such as the USA and Canada, these organizations, aligned with the theoretical proposition, adopt governance models that promote the separation of ownership and decision rights. However, in Brazil, despite the lack ofquantitative studies that measure governance separation, anecdotal evidence suggests that ownership and decisions rights are concentrated in Brazilian cooperatives.

1 Professor da FEA/RP - USP. E-mail: drmouracosta@usp.br

2 Assistant Professor. University of Missouri (USA) e Insper (Instituto de Ensino e Pesquisa). E-mail: chaddadf@missouri.edu

3 Professor da FGV-EESP. E-mail: paulo.azevedo@fgv.br 
By means of a survey with 77 Brazilian agricultural cooperatives, this paper measures the degree of de jure and de facto separation of ownership and decision control, providing new evidences on the governance models observed in different countries. Different models coexist in Brazil, i.e., although the majority of agricultural cooperatives concentrate ownership and decision rights, a significant proportion organize themselves consistently with the theoretical proposition, adopting the American models of agricultural cooperatives. Further research is required in order to address the determinants of the variety of governance models in Brazil.

Key-words: agricultural cooperatives, control and corporate governance.

Classificação JEL: D22, D23, M21, Q13.

\section{Introdução}

O instituto legal e doutrinário que rege as cooperativas agropecuárias brasileiras estabelece que o direito ao controle do proprietário é desvinculado das suas cotas de capital e do montante das transações econômicas que realiza com a organização. Essa particularidade da governança dessas organizações, aliada à propriedade difusa, presença de contratos incompletos e assimetria de informação, gera problemas de controle em cooperativas ${ }^{4}$, tais como expropriação de grupos de sócios ou consumo de bens não pecuniários.

Em países como Holanda, Suécia, Finlândia, Estados Unidos da América (EUA), entre outros, as cooperativas agropecuárias apresentam modelos de governança corporativa que desvinculam os proprietários das decisões de gestão. Isto é, a configuração comum nesses países estabelece a existência do conselho de administração eleito pelos associados para executar as decisões de controle e a contratação de executivos responsáveis pela gestão da cooperativa ${ }^{5}$ (HENDRIKSE, 2005; PELLERVO, 2000; GINDER e DEITER, 1989). No Brasil, Bialoskorski

4 Os trabalhos que abordam a problemática da governança corporativa em cooperativas para tentar explicar a sua sobrevivência sintetizam cinco desafios aos gestores: problema do carona (freerider), horizonte, portfólio, controle e custos de influência. Sugere-se ao leitor interessado na questão o texto de Cook (1995).

5 Os autores não apontam a impossibilidade de o responsável pela gestão ser cooperado da cooperativa. A característica importante consiste no conselho de administração ter direito de controle formal sobre esse executivo, que consiste em autoridade para nomear ou demitir na medida em que os contratos entre ambos não sejam cumpridos.
Neto (2003), Waack e Machado Filho (1999) e Zylbersztajn (1994), baseados em evidências pontuais sobre a presença de cooperados nos cargos de tomada de decisão, apontam que há indícios de que nessas organizações não há separação entre propriedade e controle ${ }^{6}$.

A aparente diferença entre as cooperativas agropecuárias brasileiras e aquelas localizadas em países de economia desenvolvida motivou a realização desse trabalho, que objetivou averiguar a existência da separação entre propriedade e decisão de gestão nas organizações brasileiras e avaliar se a separação ocorre de jure ou de facto.

O trabalho inova em relação aos demais por apresentar uma metodologia que permite apontar a existência da separação entre propriedade e decisão de gestão nas cooperativas agropecuárias brasileiras. O constructo é sintetizado em um índice, cujo valor permite inferir o grau de separação entre propriedade e decisões de gestão nestas organizações e ainda se a mesma ocorre de jure ou de facto.

Nas próximas quatro seções são apresentadas as etapas complementares desse artigo: o referencial teórico, a metodologia, os resultados e conclusões.

6 Uma vez que existe alguma confusão de nomenclatura sobre o conceito, conforme aponta Fama e Jensen (1983a), se infere que, para o caso brasileiro, os autores assumem a não desvinculação dos proprietários das decisões de gestão. 


\section{Referencial teórico}

A separação entre propriedade e decisão de gestão existe se os proprietários são desvinculados das decisões de gestão e é recomendada para as organizações de propriedade difusa e complexas. Ainda, a separação se concretiza se os contratos formais e as relações de agência preservam os interesses dos proprietários e gestores, isto é, garantem que o interesse dos proprietários seja mantido e que os riscos da atividade não sejam alocados aos responsáveis pela decisão de gestão (BAYSINGER e HOSKISSON, 1990). A partir de Fama e Jensen (1983), Jensen e Meckling (1976) e Alchian e Demsetz (1972), é possível estabelecer as seguintes condições para que a separação exista, ou seja, para que os detentores dos direitos aos resultados da organização sejam desvinculados das decisões de gestão:

C1. Os proprietários delegam o direito de controle formal ao conselho de administração, ou seja, lhes transferem autoridade formal sobre o controle da organização.

C2. As decisões de controle (ratificação e monitoramento) e gestão (iniciação e implantação) são alocadas respectivamente no conselho de administração e ao agente responsável pela gestão.

A condição C1 é satisfeita se o conselho administrativo tem autoridade para nomear e destituir o agente investido de autoridade para conduzir a gestão, mesmo que ele seja um dos seus membros, sem consultar os demais proprietários.

A condição C2, por sua vez, é atendida se o conselho aloca autoridade sobre as decisões de gestão para o agente definido como o principal responsável pela gestão da organização. Em contrapartida, C2 não é atendida se o presidente do conselho ou CEO assume ambos os cargos e passa, por esse motivo, a propor e ratificar os projetos de investimento da organização.

A condição $\mathrm{C} 1$ representa a condição necessária para a separação existir de jure, enquanto C2 determina se a mesma ocorre de facto.
Portanto, baseando-se na satisfação de C1 e C2, as estruturas de governança das organizações podem ser caracterizadas como separadas quase completamente, parcialmente ou concentradas ${ }^{7}$.

A partir das condições apresentadas, se estabelece que a separação entre propriedade e decisão de gestão (Y) é função da satisfação de C1 e C2, que depende da inexistência da dualidade dos ocupantes dos cargos de presidente do conselho e CEO (d) e do nível de empenho dos integrantes do conselho no monitoramento das decisões de gestão (b). Isto é: $\mathrm{Y}=\mathrm{f}(\mathrm{C} 1, \mathrm{C} 2, \mathrm{~d}, \mathrm{~b})$ dos fatores que afetam a decisão de separação entre propriedade e gestão.

A separação quase completa existe se C1 e C2 são satisfeitas, não existe dualidade (d) e existe grande empenho dos integrantes do conselho no monitoramento das decisões de gestão (b). Por exemplo, o conselho de administração é eleito, seus membros se dedicam às atividades de controle da organização e possuem autoridade para destituir o principal executivo, mesmo que ele seja um dos seus membros. A sua variação entre de jure e de facto é função da satisfação de $\mathrm{C} 1$ e C2 e dos valores de d e b. Portanto, C1, C2, $\mathrm{d}$ e b têm relação direta com o grau de separação, e b é determinante para a separação de facto. Esse tipo de separação permite as especializações que promovem a maior probabilidade de sobrevivência das organizações.

A separação parcial ocorre se somente $\mathrm{C} 1 \mathrm{ou}$ C2 é satisfeita. É importante notar que C2 pode não ocorrer de facto se existe dualidade ou se os membros do conselho de administração não se dedicam às decisões de controle.

Por fim, a concentração da propriedade e das decisões de gestão ocorre se não existe delegação dos direitos de controle e nem separação do processo decisório. Isto é, não são observadas as condições C1 e C2 e, consequentemente, toda e qualquer decisão é tomada diretamente pelos sócios em reuniões que se assemelham à assembleia geral.

7 Uma vez que a descentralização ou delegação de controle não é perfeita, os conceitos de separação e concentração completa são apenas teóricos e não são possíveis de serem observados (ARROW, 1964; SIMON, 1962). 
Diante do exposto, as organizações cooperativas, se caracterizadas como complexas, têm maior probabilidade de sucesso se o conselho de administração mantiver as decisões de controle e delegar formalmente as decisões de gestão ao agente definido como executivo da organização. Nessa situação, é configurada a separação dos proprietários da gestão. Nos casos das cooperativas em que os membros do conselho de administração são eleitos e participam diretamente das decisões de gestão é configurada a não separação dos proprietários da gestão, o que seria inadequado para o caso de cooperativas complexas (HELMBERGER, 1966).

A participação do cooperado no processo de gestão apresenta benefícios, uma vez que esse agente contribui com sua expertise nos egócios da organização. Entretanto, à medida que a cooperativa se torna complexa, a sua participação implica numa gestão "empobrecida" (HELMBERGER, 1966, p. 1431). Em outras palavras, se o cooperado, enquanto membro eleito, exercer as decisões de controle e gestão simultaneamente, a sua atuação pode prejudicar o desempenho da organização. Essa situação é agravada se configurada a afirmativa de Rodhes (1987), que aponta que os membros do conselho administrativo têm limitada diversidade de conhecimentos e habilidades para controlar a efetiva gestão das cooperativas (RODHES, 1987).

\section{Metodologia e mensuração da separação entre propriedade e gestão}

As metodologias desenvolvidas para se mensurar o grau de separação entre propriedade e controle tradicionalmente se baseiam em indicadores que apontam a proporção entre membros internos e externos no conselho de administração ou concentração de ações na mão dos acionistas. Essas medidas, como se verá a seguir, não se adequam para representar a separação entre propriedade e decisão de gestão, especificamente se o objeto de estudo são as cooperativas agropecuárias brasileiras.
Por exemplo, os trabalhos de Berle e Means (1932), Demsetz e Lehn (1985), Bebchuck (1999), Santiago-Castro e Brown (2007) utilizam índices que representam a concentração de ações dos proprietários para caracterizar a estrutura de controle das organizações que transacionam capital em bolsas. Entretanto, essa medida de concentração é imprópria para análise em cooperativas na medida em que o critério de voto adotado nessas organizações se dá majoritariamente com base no princípio do "um homem um voto" e não em voto por ações. Em outras palavras, o sistema de voto na cooperativa não é função dos direitos ao fluxo de caixa líquido ${ }^{8}$.

No que se refere à proporção entre membros internos e externos no conselho de administração, esse indicador representa uma redução na probabilidade de expropriação dos principais pelos agentes (HERMALIN e WEISBACH, 1988). Porém, essa relação não se aplica para as cooperativas brasileiras, pois o seu conselho de administração por impedimento legal é composto exclusivamente por membros eleitos.

Gorton e Schmid (1999) utilizam o número de cooperados como proxy para um índice que representa a separação entre propriedade e controle para cooperativas de crédito. Porém, uma vez que a separação é função da propriedade difusa e complexidade da organização, o indicador é definido tautologicamente, uma vez que a complexidade é causa da separação e não a própria separação. Com uma medida dessa espécie é literalmente impossível testar a hipótese de que complexidade é um determinante da separação entre controle e gestão.

Com base no exposto, antes de apresentar um índice que representa a separação, é preciso descrever uma metodologia para averiguar a sua existência nas cooperativas agropecuárias brasileiras. O método deve enfocar primariamente dois elementos: a) delegação dos direitos de controle formal, ou seja, tentar responder se a

\footnotetext{
8 Em cooperativas, o direito ao fluxo de caixa líquido ocorre com base na movimentação econômica (patronage) que o cooperado realizou junto à cooperativa no período (STAATZ, 1987).
} 
condição C1 é observada e b) alocação do processo decisório, isto é, se as decisões de controle e gestão estão separadas.

As averiguações de (a) e (b) apontam, respectivamente, a ocorrência das condições $\mathrm{C} 1$ e C2 para a separação entre propriedade e decisão de gestão e refletem as características de propriedade difusa e complexidade da cooperativa.

A averiguação de C1 é realizada tomando-se como unidade de análise o contrato da organização, que descreve como os direitos de propriedade dos sócios são alocados e exercidos. Por exemplo, o contrato ou estatuto social das organizações gera a informação sobre a alocação dos direitos de controle residual e formal entre os proprietários e conselho de administração.

A verificação de C2 é feita a partir de informações que relatam de facto como as decisões de controle e gestão são alocadas nas organizações e o nível de dedicação dos membros do conselho às decisões de controle. Portanto, a unidade de análise é a relação de agência entre conselho de administração e responsável pela gestão.

No Quadro 1 são elencadas as variáveis binárias utilizadas para representar a delegação do direito de controle formal e alocação das decisões de gestão e controle nas organizações.

A definição dessas informações é baseada em trabalhos apresentados anteriormente, que abordam o tema para organizações cooperativas e não cooperativas, tais como Williamson (2007),
Hermalin e Weisbach (2003), Baker et al. (1999), Aghion e Tirole (1997), Ginder e Deiter (1989), Fama e Jensen (1983) e Alchian e Demsetz (1972).

Hermalin e Weisbach (2003), Shleifer e Vishny (1997) e Williamson (1984), entre outros, expõem que é de responsabilidade específica do conselho de administração garantir que os agentes internos à organização não expropriem os proprietários. Para Mizruchi (1983), a manifestação desse direito culmina na autoridade formal do conselho para contratar e demitir o responsável pela gestão da empresa. Portanto, observar a quem cabe o direito formal de definir o ocupante do cargo de principal executivo $(x)$ revela se ocorre a delegação do direito de controle formal ao conselho administrativo. Em se tratando de uma variável binária, caso o órgão possua esse direito, tem-se $x_{1}=1$.

Para Fama e Jensen (1983), as atividades de proposição $\left(z_{3}\right)$ e implementação $\left(z_{4}\right)$ dos projetos devem ser alocadas aos gestores da organização. Enquanto que a ratificação $\left(z_{1}\right)$ e o monitoramento $\left(\mathrm{z}_{4}\right)$ de projetos devem ser responsabilidade do conselho de administração. Especificamente para cooperativas, Gray e Buttler (1994) e Ginder e Deiter (1989) apontam para a tendência de as decisões operacionais serem delegadas aos gerentes da organização, enquanto que o estabelecimento das políticas da sociedade e das metas a serem cumpridas é mantido no conselho diretivo.

Quadro 1. Variáveis que caracterizam a alocação dos direitos e decisões nas organizações cooperativas.

\begin{tabular}{|c|c|}
\hline $\begin{array}{l}\text { Condição C1 } \\
\text { Delegação do Direito de Controle Formal (X) }\end{array}$ & Órgão responsável \\
\hline Contratar e demitir o responsável pela gestão $(x)$ & Conselho de Administração \\
\hline $\begin{array}{l}\text { Condição C2 } \\
\text { Alocação das Decisões de Gestão e Controle (Z) }\end{array}$ & Órgão responsável \\
\hline ·Decisões de Controle & \multirow{3}{*}{ Conselho de Administração } \\
\hline Ratificar os projetos de investimentos $\left(z_{1}\right)$ & \\
\hline Monitorar o desempenho da gestão $\left(z_{2}\right)$ & \\
\hline ·Decisões de Gestão & \multirow{3}{*}{ Agente responsável pela gestão } \\
\hline Propor projetos $\left(z_{3}\right)$ & \\
\hline Implementar projetos $\left(z_{4}\right)$ & \\
\hline
\end{tabular}


Por meio das informações coletadas a campo é possível definir um índice de separação entre propriedade e decisão de gestão $(\mathrm{Y})$, apresentado abaixo, que caracteriza a estrutura de governança da cooperativa.

$$
\mathrm{Y}=\mathrm{X}+\mathrm{Z}, \mathrm{Y} \in[0,2]
$$

O índice (Y) é composto por dois elementos. $\mathrm{O}$ primeiro $(\mathrm{X})$ representa como os direitos de controle residual e formal estão alocados na organização, ou seja, se a condição C1 é satisfeita. Por exemplo, se os direitos de controle residual e formal estão alocados respectivamente nos proprietários e conselho de administração, tem-se que C1 é atendida e, portanto, $\mathrm{X}=1$.

$O$ segundo elemento $(Z)$, definido conforme equação (2) abaixo, é refletido na relação de agência entre conselho de administração e o responsável pela gestão. A observação gera informações sobre C2.

$\mathrm{Z}=\mathrm{f}\left(\mathrm{z}_{\mathrm{i}} ; \mathrm{b} ; \mathrm{d}\right)=\mathrm{d} *\left[\frac{\left(\mathrm{z}_{\mathrm{PR}}+\mathrm{z}_{\mathrm{IM}}\right)}{2} * \mathrm{~b}\right]$,

tal que $0 \leq \mathrm{Z} \leq 1$

Na equação (2) as variáveis $\mathrm{Z}_{\mathrm{PR}}$ e $\mathrm{Z}_{\mathrm{IM}}$ são dummies criadas a partir da observação de como as alocações das decisões de controle e gestão ocorrem e demonstram a sua separação ou concentração na cooperativa. As variáveis representam, respectivamente, como os projetos são propostos e ratificados e implementados e monitorados e seus valores são definidos considerando-se as seguintes proposições adiante:

$$
\begin{aligned}
& \mathrm{Z}_{\mathrm{PR}}=\left\{\begin{array}{l}
1=, \text { se } \mathrm{Z}_{1}=\mathrm{z}_{3} \\
0=, \text { se } \mathrm{Z}_{1} \neq \mathrm{Z}_{3}
\end{array}\right. \\
& \mathrm{Z}_{\mathrm{IM}}=\left\{\begin{array}{l}
1=, \text { se } \mathrm{z}_{2}=\mathrm{z}_{4} \\
0=, \text { se } \mathrm{Z}_{2} \neq \mathrm{Z}_{4}
\end{array}\right.
\end{aligned}
$$

Quando $z_{1} \neq z_{3}$, tem-se que a proposição $\left(z_{3}\right)$ e ratificação de projetos $\left(z_{1}\right)$ estão concentradas no principal ou agente; caso contrário, estariam separadas. Da mesma forma, se $\mathrm{z}_{2} \neq \mathrm{z}_{4}$, as atividades de monitoramento $\left(z_{2}\right)$ e implantação $\left(z_{4}\right)$ de projetos são concentradas em uma única parte da relação de agência. Essas hipóteses implicam numa relação positiva da informação $z_{i}$ com o grau de separação entre propriedade e decisão de gestão.
O valor de $\left(\frac{Z_{P R}+Z_{I M}}{2}\right)$ é ponderado por $b$, calculado conforme apontado em (3), que representa o nível de dedicação dos membros do conselho de administração ao processo decisório da cooperativa, que, conforme Aghion e Tirole (1997), é uma medida da separação entre autoridade formal e autoridade real (ou de facto). Por simplicidade, o índice considera apenas o tempo que o presidente do conselho se dedica à cooperativa.

$$
\mathrm{b}=\frac{\mathrm{h}}{\mathrm{H}} \text {, tal que } 0<\mathrm{b} \leq 1
$$

$\mathrm{O}$ b é definido como a relação entre o total de horas (h) que o presidente do conselho de administração se dedica às atividades da cooperativa e o total de horas semanais $(\mathrm{H})$ de expediente da organização. Na medida em que o presidente aumenta ou diminui suas horas de dedicação (h) na organização tem-se, respectivamente, que $b \rightarrow 1$ ou $b \rightarrow 0$. Esse esforço é assumido como determinante para seu conjunto de informações sobre a gestão da organização9.

De acordo com Staatz (1987), as cooperativas agropecuárias são sociedades que afetam diretamente o nível de renda dos proprietários, de tal modo que é necessário maior intensidade de monitoramento do conselho sobre o principal executivo nesse tipo de sociedade (HUETH e MARCOUL, 2008). Portanto, $b$ afeta direta e positivamente o grau de separação entre propriedade e decisão de gestão das cooperativas. Assim, na medida em que $b \rightarrow 1$, o presidente tende a desempenhar de facto as atividades de ratificação e monitoramento ${ }^{10}$.

Adicionalmente, a variável $\mathrm{d}$, em (2), é binária e revela se o cargo de agente responsável pela gestão é ocupado simultaneamente pelo

9 Em (3) é estabelecido $\mathrm{H}=48$ horas, que representa seis dias de expediente semanais com oito horas diárias. Adicionalmente, é assumido que a dedicação do presidente à cooperativa sempre será positiva e não nula $(b>0)$.

10 Importante observar que, se o nível de esforço do presidente do conselho for direcionado exclusivamente para as atividades de gestão, a sua atuação na organização poderá afetar o grau de delegação de autoridade real do agente sobre as decisões de gestão. Essa concentração de autoridade terá reflexos no desempenho da organização. 
presidente do conselho de administração, situação denominada na literatura por 'dualidade'. Caso haja dualidade, tem-se $\mathrm{d}=0$. Portanto, o valor $\mathrm{Z}$ indica se a cooperativa apresenta separação das decisões de controle e gestão, que pode variar entre inexistente, parcial ou completa. Caso $Z=0$, é inexistente; se $Z$ é tal que $0<Z \leq 0,5$, a separação é parcial; e com $Z>0,5$,esta é completa ${ }^{11}$.

A partir dos valores de $\mathrm{X}$ e $\mathrm{Z}$ tem-se o cálculo de $Y$. Se $Y=0$, C1 e C2 não são satisfeitas, o que caracteriza uma estrutura de propriedade que não desvincula os proprietários das decisões de gestão. Para os casos em que o valor de $\mathrm{Y}$ é tal que $0<\mathrm{Y} \leq 1$, o arranjo de governança apresenta separação parcial, caracterizado pela existência somente de $\mathrm{C} 1$ ou $\mathrm{C} 2$.

Os valores do índice tal que $1<\mathrm{Y} \leq 1,5$ caracterizam a separação como quase completa de jure. Essa estrutura de propriedade demanda que $\mathrm{C} 1$ seja satisfeita; $\mathrm{z}_{\mathrm{PR}}$ ou $\mathrm{z}_{\mathrm{IM}}=1 \mathrm{e} b>0$. Nos casos em que $Y>1,5$, a separação é quase completa de facto e demonstra que a alocação do direito de controle formal ocorre e as decisões de controle e gestão são alocadas respectivamente no conselho de administração e agente responsável pela gestão de facto. Para que se concretize a separação de facto, assume-se que, nesse tipo de estrutura de governança, as condições C1 e C2 sejam plenamente satisfeitas com $b>0,5$.

\subsection{Amostra e coleta de dados}

As cooperativas que compuseram a amostra foram as singulares, centrais ou federações atuantes em atividades vinculadas ao agronegócio brasileiro, localizadas nos estados de Minas Gerais, Paraná, Rio Grande do Sul, Santa Catarina e São Paulo. Os estados foram escolhidos por congregarem conjuntamente 702 organizações, quantidade equivalente a $45 \%$ do total das

$11 \mathrm{O}$ valor $\mathrm{Z}=0,5$ é estabelecido para garantir que as decisões de controle e gestão, representadas pelo valor de $\left(\frac{Z_{\mathrm{PR}}+\mathrm{Z}_{\mathrm{IM}}}{2}\right)$, sejam ao menos parcialmente separadas, e que o presidente do conselho dedique no mínimo $50 \%$ do seu tempo às atividades da cooperativa. Importante ainda notar que existe $Z \neq 0$ se e somente se $d=1,\left(\frac{Z_{P R}+Z_{I M}}{2}\right)$ e $b>0$. cooperativas similares em todo País e acima de $90 \%$ do total faturado em 2008. A Tabela 1 aponta a amostragem por unidades da federação.

Conforme apontado na metodologia, a pesquisa de campo consistiu em coletar informações primárias junto às cooperativas sobre delegação dos direitos de controle formal, alocação das decisões de controle e gestão, nível de dedicação do presidente e dualidade do cargo de agente responsável pela gestão. Essas informações foram retiradas dos seus estatutos sociais e das respostas obtidas no questionário encaminhado ao presidente do conselho de administração.

Os estatutos sociais foram acessados nas sedes das unidades estaduais da Organização das Cooperativas Brasileiras $(\mathrm{OCB})^{12}$. A exceção ocorreu no estado de São Paulo, onde o contato foi feito diretamente com os presidentes das cooperativas e solicitado o encaminhamento do documento para análise.

A fim de se ter uma amostra representativa, foi estabelecida uma amostra mínima de 25\% do total de cooperativas existentes em 2008 para cada estado. Adicionalmente, para garantir uma amostra aleatória, foram escolhidos os estatutos com base no número de registro da cooperativa na organização de representação estadual ou por seu município sede. Esse procedimento evitou que o pesquisador tivesse contato com o nome da cooperativa e que isso orientasse a escolha. A Tabela 2 apresenta o total de estatutos analisados por estado e representatividade da amostra.

\footnotetext{
12 A Lei n. 5.764/71 estabelece a existência da Organização das Cooperativas Brasileiras (OCB), que representa, em termos nacionais, as cooperativas brasileiras, e é constituída de uma unidade para cada estado - Organização das Cooperativas Estaduais (OCE).
} 
Tabela 1. Distribuição das cooperativas agropecuárias nos estados definidos para estudo - 2008.

\begin{tabular}{lcccc}
\hline \multirow{2}{*}{ Estado } & \multicolumn{2}{c}{ Cooperativas } & \multicolumn{2}{c}{ Faturamento } \\
\cline { 2 - 5 } & Total & $\%$ & Total (em bilhões - R\$) & $\%$ \\
\hline Minas Gerais & 192 & 12 & 13,87 & 19 \\
Paraná & 80 & 5 & 18,60 & 26 \\
Rio Grande do Sul & 242 & 16 & 9,64 & 13 \\
Santa Catarina & 54 & 3 & 8,07 & 11 \\
São Paulo & 134 & 9 & 15,32 & 21 \\
Amostra & 702 & 45 & 65,5 & 91 \\
Brasil & 1544 & 100 & 72,2 & 100 \\
\hline
\end{tabular}
Fonte: OCB (2009).

Tabela 2. Distribuição da amostra de cooperativas acessadas na pesquisa.

\begin{tabular}{lcccccc}
\hline \multirow{2}{*}{\multicolumn{1}{c}{ Estado }} & \multicolumn{2}{c}{ Estatutos avaliados } & \multicolumn{2}{c}{ Questionários respondidos } & \multicolumn{2}{c}{ Importância econômica (R\$ bi) } \\
\cline { 2 - 7 } & Total & $\%$ & Total & $\%$ & Total & $\%$ \\
\hline Minas Gerais & 60 & 31,2 & 33 & 17,2 & 3,2 & 23 \\
Paraná & 43 & 53,7 & 9 & 11,3 & 9,2 & 49 \\
Rio Grande do Sul & 60 & 24,7 & 13 & 5,4 & 2,6 & 27 \\
Santa Catarina & 35 & 64,8 & 11 & 20,4 & 2,4 & 30 \\
São Paulo & 22 & 16,4 & 11 & 8,2 & 1,9 & 12 \\
Amostra & 224 & 31,9 & 77 & 11 & 19,3 & 29 \\
\hline
\end{tabular}

A análise dos estatutos gerou informações referentes à alocação dos direitos de controle residual e formal entre proprietários e conselho de administração. O entendimento da alocação das decisões de controle e gestão e da atuação do presidente do conselho foi realizado a partir das informações coletadas por meio de questionário submetido à cooperativa ${ }^{13}$.

A construção do questionário consistiu em quatro etapas complementares: discussão teórica sobre o tipo de informação que se deveria coletar; debate com os profissionais das OCEs sobre a existência das informações solicitadas e o método de aplicação que apresentaria melhor resultado; aplicação piloto $^{14}$ in loco do questionário junto a

$13 \mathrm{O}$ fato de o questionário ter sido submetido à cooperativa para preenchimento não garante que o presidente do conselho de administração o tenha respondido e isso pode ser considerado um dos limites da pesquisa, haja visto não ter sido possível controlar quem seria o responsável pelo preenchimento da pesquisa.

14 Para realizar a aplicação piloto, foram escolhidas cooperativas com base no seu faturamento. Foi escolhida uma cooperativa com receita acima de R\$ 500 milhões, outra entre R\$ 100 e 500 milhões e ainda uma terceira, com diferentes cooperativas agropecuárias localizadas nos estados de MG e SP; e, por fim, o ajuste final na forma de indagação das questões.

O banco de dados foi construído a partir das informações retiradas do estatuto e do questionário. Primeiramente foram compilados os dados dos 224 estatutos sociais analisados e, na sequência, os dados dos 77 questionários recebidos. Na medida em que os questionários continham 40 diferentes tipos de informações, foi necessário, para alguns casos, contatar novamente a cooperativa e averiguar inconsistências ou falta de informações. Para que a cooperativa compusesse a amostra final era necessário que seu estatuto tivesse sido analisado e que respondesse o questionário. Assim, a amostra final consistiu de 77 organizações cooperativas: 74 singulares, duas centrais e uma federação. Esse número equivaleu

faturamento abaixo de $\mathrm{R} \$ 100$ milhões. O experimento consistiu em aplicar o questionário pessoalmente para averiguar os tipos de dúvidas que cada pergunta suscitaria, averiguar o número de funcionários que o presidente do conselho necessitaria consultar para responder e o tempo dispendido para o preenchimento. 
a aproximadamente $11 \%$ do total do número de cooperativas existentes nos estados e a $29 \%$ do total de faturamento.

A partir da averiguação do estatuto da cooperativa, é apontado o valor de X: se o direito de admitir e demitir o principal responsável pela gestão está alocado ao conselho de administração, tem-se $X=1$; nos casos em que o estatuto social estabelece que a assembleia geral eleja diretamente o presidente ou membros do conselho de administração para exercer as funções executivas da cooperativa, o direito de controle formal permanece com os proprietários reunidos em assembleia, de tal modo que $X=0$. Nessas situações, tem-se que C1 não é observada.

A partir dos questionários é possível averiguar as proxies das decisões de controle na cooperativa sugeridas no Quadro 1, pois se observa o responsável pela ratificação dos projetos de investimento $\left(z_{1}\right)$ e monitoramento do desempenho da gestão $\left(z_{2}\right)$, que seguem diretamente as sugestões de Fama e Jensen (1983). É importante ressaltar que, para $\left(z_{2}\right)$, não foi questionado diretamente quem monitora $\mathrm{o}$ desempenho da gestão, mas quem tem autoridade para definir a remuneração do principal responsável pela gestão da organização. Sem perda de generalidade, essa opção foi feita para evitar confusões relativas ao papel do conselho fiscal na cooperativa. Caso essas atividades estejam alocadas ao conselho de administração, tem-se que $z_{1}=z_{2}=1 \mathrm{e}$, em caso contrário, $z_{1}=$ $z_{2}=0$.

Seguindo as observações de Ginder e Deiter (1989) sobre as responsabilidades dos executivos das cooperativas e as proposições de Fama e Jensen (1983) sobre as decisões de gestão, foi averiguado quem seria o responsável por propor abertura de novas filiais $\left(z_{3}\right)$ e pela execução dos projetos para aumentar a produtividade da cooperativa $\left(\mathrm{z}_{4}\right)$. Essasinformações foram tomadas como proxies para apontar como as decisões de gestão são alocadas: quem é responsável pela proposição $\left(z_{3}\right)$ e implementação de projetos $\left(z_{4}\right)$. Caso as atividades estejam alocadas ao principal executivo, tem-se que $z_{3}=z_{4}=1$.

\section{Resultados e discussões}

As estatísticas descritivas, com as características das cooperativas da amostra,são descritas na Tabela 3. É possível averiguar que, em 2008, a cooperativa padrão da amostra tinha 37 anos, contava com 2.648 cooperados e 447 funcionários.

As cooperativas faturaram R 251,2 milhões, em média, e atuavam em cerca de sete municípios com a presença de ao menos um funcionário em tempo integral. A maior parte delas (32\%) atuava na compra, produção ou distribuição de insumos, $62 \%$ detinham algum tipo de atividade agroindustrial e, na média, apresentavam elevada concentração de atividades econômicas ${ }^{15}$.

Os proprietários (cooperados) das cooperativas na amostra se reuniram, em média uma vez a cada 197 dias em assembleia geral, ou seja, um monitoramento com mais de uma reunião por ano e um sistema de voto predominantemente (90\%) de um homem, um voto.

O conselho de administração da amostra era composto de oito membros, eleitos para um mandato de 3,2 anos e se reuniam a cada 28 dias, em média. Ainda, 71\% desses órgãos contavam com a colaboração de auditores externos, e seus presidentes dedicavam em torno de 29 horas por semana à cooperativa.

Um olhar sobre os dados das cooperativas por estado da Federação pesquisado permite apontar que no Rio Grande do Sul estavam as cooperativas com idade média mais elevada e com maior número de cooperados. No Paraná estavam as cooperativas com maior número de funcionários, que apresentaram maior faturamento por organização, maior abrangência geográfica e a maior proporção de cooperativas com atividade agroindustrial.

\footnotetext{
15 A média do índice de concentração de atividades econômicas era de 0,513 (ou 5.130 pontos), o que significa que, em média, apenas duas atividades econômicas representam quase a totalidade do faturamento das cooperativas. Este indicador foi baseado no Índice de Herfindahl Hirschmann (HHI), adaptado para mensurar o grau de concentração dos diferentes produtos no faturamento na cooperativa (SERIGATI et al., 2009).
} 
Tabela 3. Características das cooperativas agropecuárias brasileiras, consideradas as amostras e os estados amostrados (ano - 2008).

\begin{tabular}{|c|c|c|c|c|c|c|}
\hline \multirow[b]{2}{*}{ Caracterização das cooperativas } & \multicolumn{6}{|c|}{ Resultados observados } \\
\hline & $\begin{array}{l}\text { Minas } \\
\text { Gerais }\end{array}$ & Paraná & Rio Grande do Sul & Santa Catarina & São Paulo & Total \\
\hline \multicolumn{7}{|l|}{ Idade em anos } \\
\hline \multirow{2}{*}{ Média Desvio padrão } & 34 & 41 & 46 & 28 & 43 & 37 \\
\hline & 19 & 16 & 18 & 16 & 22 & 19 \\
\hline \multicolumn{7}{|l|}{ Total de cooperados } \\
\hline \multirow{2}{*}{ Média Desvio padrão } & 1408 & 3964 & 4444 & 2372 & 3442 & 2648 \\
\hline & 2270 & 7024 & 3567 & 4338 & 5367 & 4143 \\
\hline \multicolumn{7}{|l|}{ Total de funcionários } \\
\hline \multirow{2}{*}{ Média Desvio padrão } & 142 & 2012 & 342 & 378 & 274 & 447 \\
\hline & 346 & 2382 & 358 & 513 & 433 & 1031 \\
\hline \multicolumn{7}{|l|}{ Faturamento (R\$ milhões) } \\
\hline \multirow{2}{*}{ Média Desvio padrão } & 97,17 & 1018,7 & 201,7 & 220,7 & 174,3 & 251,20 \\
\hline & 301 & 1433 & 206 & 315 & 273 & 604 \\
\hline \multicolumn{7}{|l|}{ Área de atuação (municípios) } \\
\hline \multirow{2}{*}{ Média Desvio padrão } & 3 & 15 & 7 & 10 & 7 & 7 \\
\hline & 4 & 18 & 6 & 16 & 10 & 10 \\
\hline Principal atividade & Leite & Grãos & Grãos & Insumos & Insumos & Insumos \\
\hline Representatividade (\%) & 42 & 44 & 54 & 45 & 55 & 32 \\
\hline$\%$ de cooperativas com Agroindustrial & 39 & 89 & 85 & 82 & 64 & 62 \\
\hline \multicolumn{7}{|l|}{ Índice de concentração (HHI) } \\
\hline \multirow{2}{*}{ Média Desvio padrão } & 0,561 & 0,376 & 0,401 & 0,486 & 0,646 & 0,513 \\
\hline & 0,237 & 0,280 & 0,271 & 0,298 & 0,333 & 0,279 \\
\hline \multicolumn{7}{|l|}{ Periodicidade das assembleias gerais } \\
\hline \multirow{2}{*}{ Média Desvio padrão } & 214 & 196 & 191 & 167 & 193 & 197 \\
\hline & 509 & 466 & 439 & 408 & 354 & 450 \\
\hline \multicolumn{7}{|l|}{ Tipo de voto em assembleia geral } \\
\hline $\begin{array}{l}\% \text { de cooperativas com modelo um } \\
\text { homem, um voto }\end{array}$ & 97 & 67 & 92 & 82 & 91 & 90 \\
\hline \multicolumn{7}{|l|}{$\begin{array}{l}\text { Tamanho do conselho de } \\
\text { administração }\end{array}$} \\
\hline \multirow{2}{*}{ Média Desvio padrão } & 7 & 10 & 11 & 8 & 7 & 8 \\
\hline & 3 & 5 & 5 & 3 & 5 & 4 \\
\hline \multicolumn{7}{|l|}{ Mandato (anos) } \\
\hline \multirow{2}{*}{ Média Desvio padrão } & 3,2 & 3,5 & 2,9 & 3,4 & 3,5 & 3,2 \\
\hline & 0,6 & 0,5 & 0,6 & 0,7 & 0,5 & 0,6 \\
\hline \multicolumn{7}{|l|}{$\begin{array}{l}\text { Periodicidade das reuniões do } \\
\text { conselho(dias) }\end{array}$} \\
\hline \multirow{2}{*}{ Média Desvio padrão } & 29 & 26 & 28 & 24 & 29 & 28 \\
\hline & 121 & 88 & 106 & 29 & 78 & 65 \\
\hline \multicolumn{7}{|l|}{$\begin{array}{l}\text { Dedicação do Presidente do conselho } \\
\text { (em horas) }\end{array}$} \\
\hline \multirow{2}{*}{ Média Desvio padrão } & 22 & 31 & 43 & 37 & 25 & 29 \\
\hline & 17 & 21 & 3 & 13 & 15 & 17 \\
\hline$\%$ de cooperativas com auditoria externa & 55 & 78 & 85 & 91 & 82 & 71 \\
\hline
\end{tabular}


No Rio Grande do Sul, as cooperativas atuavam predominantemente na produção de grãos (soja), enquanto que, no Paraná, mesmo com a maior parte das cooperativas atuando na mesma área, há uma menor concentração de atividades econômicas, haja visto o seu índice $\mathrm{HHI}$ ter sido o menor entre todos os estados. Em Santa Catarina, os cooperados se reuniram com mais frequência que nos demais estados; no Paraná, as cooperativas proporcionalmente possuem mais modelos de votação que diferem do princípio um homem, um voto.

As cooperativas com maior número de integrantes no conselho de administração eram as do Rio Grande do Sul, enquanto que as de São Paulo e Minas Gerais eram as com menor quantidade de integrantes. Os membros dos conselhos que se reuniam com menor intervalo eram os de Santa Catarina, que também eram os que mais contavam com a colaboração de auditores externos.Os membros do conselho administrativo das cooperativas gaúchas tinham o menor tempo de mandato entre os demais estados e, ao mesmo tempo, os seus presidentes, em média, se dedicaram mais às atividades da organização.

\subsection{Separação entre propriedade e decisões de gestão de jure}

A averiguação da satisfação de $\mathrm{C} 1$ - ou seja, da separação de jure - foi realizada via avaliação dos estatutos sociais das cooperativas. Porém, dada a elevada concentração do número de presidentes dos conselhos (62\%) que assumiram as funções de executivo, foi necessário complementar a análise considerando as respostas coletadas a campo. Assim, nos estatutos, foi avaliado como se dá a alocação do direito de definir o presidente do conselho de administração e, a partir do questionário, foi investigado se o agente responsável pela gestão ocupava simultaneamente o cargo de presidente do conselho. Essas informações foram contrastadas para diagnosticar se o conselho mantinha o direito de nomear ou destituir o agente responsável por conduzir as decisões de gestão, mesmo que este fosse membro do conselho. Os resultados são apontados na Tabela 4.

O contraste apontou que, nos casos em que o agente responsável pela gestão era presidente do conselho e o órgão não tinha autoridade para destituí-lo ou nomeá-lo, a separação não existia de

Tabela 4. Delegação do direito de controle formal ou separação de jure nas cooperativas agropecuárias brasileiras.

\begin{tabular}{|c|c|c|c|c|c|c|}
\hline \multirow{3}{*}{ Alocação do direito de controle formal } & \multicolumn{6}{|c|}{ Resultados observados } \\
\hline & \multicolumn{2}{|c|}{ Minas Gerais } & \multicolumn{2}{|c|}{ Paraná } & \multicolumn{2}{|c|}{ Rio Grande do Sul } \\
\hline & Qtd. & $\%$ & Qtd. & $\%$ & Qtd. & $\%$ \\
\hline Cooperativas com $\mathrm{d}=0$ & 22 & 67 & 5 & 56 & 8 & 62 \\
\hline Cooperativas com $X=1$ & 16 & 48 & 4 & 44 & 1 & 8 \\
\hline Cooperativas com $(X=1) \cap(d=0)$ & 5 & 31 & 3 & 75 & 0 & 0 \\
\hline Cooperativa com $(X=1) \cap(\mathrm{d}=1)$ & 11 & 69 & 1 & 25 & 1 & 100 \\
\hline Satisfação da Condição C1 - (X) & 16 & 48 & 4 & 44 & 1 & 8 \\
\hline \multirow{3}{*}{ Alocação do direito de controle formal } & \multicolumn{6}{|c|}{ Resultados observados } \\
\hline & \multicolumn{2}{|c|}{ Santa Catarina } & \multicolumn{2}{|c|}{ São Paulo } & \multicolumn{2}{|c|}{ Total } \\
\hline & Qtd. & $\%$ & Qtd. & $\%$ & Qtd. & $\%$ \\
\hline Cooperativas com $\mathrm{d}=0$ & 5 & 45 & 7 & 64 & 47 & 62 \\
\hline Cooperativas com $X=1$ & 2 & 18 & 6 & 55 & 29 & 38 \\
\hline Cooperativas com $(X=1) \cap(d=0)$ & 0 & 0 & 4 & 67 & 12 & 41 \\
\hline Cooperativa com $(X=1) \cap(d=1)$ & 2 & 100 & 2 & 33 & 17 & 59 \\
\hline Satisfação da Condição C1 - (X) & 2 & 18 & 6 & 55 & 29 & 38 \\
\hline
\end{tabular}


jure. Entre as cooperativas da amostra, apenas 38\% delas recebem dos cooperados o direito de controle formal. Em outras palavras, recebem autoridade para definir quem será o executivo responsável pela gestão. Portanto, nessas cooperativas, a condição C1 não é satisfeita, pois o direito de controle formal é mantido nos cooperados reunidos em assembleia geral e não nos membros do conselho de administração.

O cargo de principal executivo foi ocupado pelo presidente do conselho de administração em $62 \%$ das cooperativas amostradas. Desta quantidade, $74 \%$ foram eleitos diretamente pelos cooperados reunidos em assembleia geral e $26 \%$ foram nomeados pelo conselho de administração. Entre os conselhos com autoridade para nomear o executivo, apenas $41 \%$ nomearam membros do conselho.

Minas Gerais foi o estado com maior presença de membros do conselho no cargo de agente responsável pela gestão, enquanto São Paulo concentrava o maior número de conselhos com autonomia para nomear o executivo. Na situação oposta, Santa Catarina era o que tinha menor presença de membros do conselho ocupando o cargo de agente responsável pela gestão, e Rio Grande do Sul foi o estado cujo cooperado delega menos direito de controle formal aos conselhos.

\subsection{Separação entre propriedade e decisões de gestão de facto}

A verificação de C2 foi feita a partir de informações coletadas a campo, a fim de relatar como as decisões de controle e gestão estão alocadas nas organizações. Essas informações e o nível de dedicação do presidente do conselho às atividades da cooperativa (b) e existência de dualidade (d) foram então reunidos na equação (2), que sintetiza o constructo sobre a separação entre as decisões de controle e gestão.

Uma visão sobre a alocação das decisões de controle e gestão, separadas por estado, é apresentada na Tabela 5 a seguir. A sua apreciação permite averiguar a porcentagem de cooperativas que apresentaram alocação de decisão conforme é estabelecido no tópico de referencial teórico.

Tabela 5. Separação das decisões de controle e gestão nas cooperativas agropecuárias brasileiras (ano - 2008).

\begin{tabular}{|c|c|c|c|c|c|c|}
\hline \multirow{2}{*}{$\begin{array}{l}\text { Separação das decisões de controle } \\
\text { e gestão nas cooperativas }\end{array}$} & \multicolumn{6}{|c|}{ Resultados observados (\%) } \\
\hline & Minas Gerais & Paraná & $\begin{array}{c}\text { Rio Grande } \\
\text { do Sul }\end{array}$ & Santa Catarina & São Paulo & Total \\
\hline Cooperativas com $z_{1}=1$ & 85 & 78 & 85 & 73 & 91 & 83 \\
\hline Cooperativas com $z_{2}=1$ & 42 & 56 & 38 & 55 & 55 & 47 \\
\hline Cooperativas com $z_{3}=1$ & 30 & 56 & 61 & 36 & 36 & 40 \\
\hline Cooperativas com $z_{4}=1$ & 61 & 89 & 92 & 54 & 64 & 69 \\
\hline Cooperativas com $\mathrm{Z}_{\mathrm{PR}}=1$ & 45 & 33 & 77 & 45 & 45 & 49 \\
\hline Cooperativas com $\mathrm{z}_{\mathrm{MI}}=1$ & 64 & 44 & 46 & 45 & 54 & 54 \\
\hline Cooperativas com $\left(\mathrm{z}_{\mathrm{PR}}=1\right) \cap\left(\mathrm{z}_{\mathrm{IM}}=1\right)$ & 30 & 22 & 31 & 9 & 36 & 27 \\
\hline Cooperativas com $\left(\mathrm{Z}_{\mathrm{PR}}=0\right) \cap\left(\mathrm{Z}_{\mathrm{IM}}=0\right)$ & 21 & 44 & 8 & 18 & 36 & 23 \\
\hline Cooperativas com $\left(\mathrm{Z}_{\mathrm{PR}}=1\right) \cap\left(\mathrm{Z}_{\mathrm{IM}}=0\right)$ & 15 & 11 & 46 & 36 & 9 & 28 \\
\hline Cooperativas com $\left(\mathrm{Z}_{\mathrm{PR}}=0\right) \cap\left(\mathrm{Z}_{\mathrm{IM}}=1\right)$ & 33 & 22 & 15 & 36 & 18 & 22 \\
\hline \multicolumn{7}{|l|}{ Satisfação da condição C2: } \\
\hline Cooperativas com não separação $(Z=0)$ & 70 & 67 & 62 & 55 & 64 & 65 \\
\hline $\begin{array}{l}\text { Cooperativas com separação parcial } \\
(0<Z \leq 0,5)\end{array}$ & 24 & 33 & 15 & 45 & 27 & 27 \\
\hline $\begin{array}{l}\text { Cooperativas com separação completa } \\
(Z>0,5)\end{array}$ & 6 & 0 & 23 & 0 & 9 & 8 \\
\hline Valor médio de $Z$ & 0,09 & 0,06 & 0,27 & 0,14 & 0,11 & 0,13 \\
\hline Desvio padrão & 0.22 & 0,13 & 0,38 & 0,18 & 0,17 & 0,24 \\
\hline
\end{tabular}


Os dados apontam que apenas $8 \%$ das cooperativas da amostra separam as decisões de controle e gestão completamente, isto é, o conselho de administração exerce as atividades de ratificação dos projetos e monitora a sua execução, e o agente responsável pela gestão executa as funções de proposição e implementação dos projetos. Cerca de 30\% das organizações apresentam separação parcial, isto é, parte das atividades é alocada e parte, concentrada.

A maior parte das organizações $(65 \%)$ não apresenta separação alguma no processo decisório, e a razão preponderante é o acúmulo das funções de presidente do conselho e agente responsável pela gestão. Nessa situação, os membros do conselho atuam, por exemplo, como propositor e ratificador dos projetos de investimento, descaracterizando a desejável separação entre controle e gestão de organizações difusas e complexas.

O Rio Grande do Sul é a unidade da federação com maior proporção de cooperativas que separam completamente o processo decisório (23\%). Em contrapartida, Minas Gerais é o estado onde a concentração das decisões de controle e gestão ocorre com maior frequência (70\%); entre os estados cujascooperativas apresentam separação parcial, Santa Catarina é o estado que concentra a maior parte das cooperativas nessa categoria. Um olhar apenas sobre as decisões de controle e gestão permite apontar que $83 \%$ das cooperativas alocam a decisão sobre ratificar os projetos de investimento ao conselho de administraçãoe $47 \%$ lhe delega a responsabilidade de definir a remuneração do agente responsável pela gestão. Em se tratando das decisões de gestão, 40\% das organizações analisadas alocam as responsabilidades de proposição de projetos aos executivos e $69 \%$ lhes delega autoridade para implementar os projetos aprovados.

Ainda de forma parcial, os valores de $\mathrm{z}_{\mathrm{PR}}$ e $\mathrm{Z}_{\mathrm{IM}}$ revelam que $49 \%$ das cooperativas têm as atividades de proposição e ratificação de projetos separadas. Isto é, não estão concentradas no conselho ou no responsável pela gestão. Da mesma forma, $54 \%$ delas não têm as atividades de implantação e monitoramento concentradas.

\subsection{Separação entre propriedade e decisões de gestão de jure e de facto}

Os valores do índice (Y), que representa a separação entre propriedade e as decisões de gestão de jure ou de facto, definido conforme (1), é apresentado na Tabela 6 .

A partir da análise da tabela nota-se que, entre as cooperativas da amostra, o valor médio de $Y$ era 0,51 , o que equivale a existência de separação parcial. Ou seja, embora haja grande variação nos índices, as cooperativas brasileiras, em média, delegam o direito de controle formal ao conselho de administração ou separam as decisões de gestão e controle. Porém, não fazem ambos simultaneamente.

Tabela 6. Separação entre propriedade e decisões de gestão nas cooperativas agropecuárias brasileiras amostradas (ano-2008).

\begin{tabular}{lcccccc}
\hline \multicolumn{1}{c}{$\begin{array}{c}\text { Índice de separação entre } \\
\text { propriedade e gestão }\end{array}$} & \multicolumn{5}{c}{ Dados por estado da amostra } \\
\cline { 2 - 7 } & Minas Gerais & Paraná & Rio Grande do Sul & Santa Catarina & São Paulo & Total \\
\hline Índice de separação médio & 0,58 & 0,51 & 0,35 & 0,32 & 0,66 & 0,51 \\
Desviopadrão & 0,64 & 0,50 & 0,59 & 0,45 & 0,52 & 0,57 \\
$\%$ de cooperativas com $(\mathrm{Y}=0)$ & 52 & 33 & 62 & 55 & 27 & 48 \\
$\%$ de cooperativas com $(0<\mathrm{Y} \leq 1)$ & 18 & 56 & 31 & 27 & 55 & 31 \\
$\%$ de cooperativas com $(\mathrm{Y}>1)$ & 30 & 11 & 0 & 18 & 18 & 21 \\
\multicolumn{1}{c}{ de jure $(1<\mathrm{Y} \leq 1,5)$} & 24 & 11 & 0 & 0 & 18 & 17 \\
de facto $(\mathrm{Y}>1,5)$ & 6 & 0 & & 0 & 4 \\
\hline
\end{tabular}


Adicionalmente, nota-se que, entre as cooperativas da amostra, $48 \%$ não possuíam separação de jure e de facto. Em outras palavras, sua estrutura de governança não desvinculava os proprietários das decisões de gestão, haja vista a não satisfação das condições $\mathrm{C} 1$ e C2. Portanto, nessas organizações, o conselho de administração não detém autoridade para nomear ou destituir o agente responsável pelas decisões de gestão e as decisões de controle e gestão não são separadas.

Ainda, 31\% das cooperativas amostradas apresentaram separação parcial, ou seja, somente $\mathrm{C} 1$ ou C2 foi satisfeita. Entre essas cooperativas, $44 \%$ não satisfizeram $\mathrm{C} 1$ e o restante (56\%) não contemplaram a condição C2. Assim, as primeiras não delegaram ao conselho a autoridade para definir o agente responsável pela gestão e as demais não separavam as decisões de controle e gestão. Entre as cooperativas que não satisfizeram C2, em apenas $6 \%$ tal fato decorria exclusivamente da não separação das decisões de controle e gestão. Em cerca de dois terços dos casos, a não satisfação de C2 ocorria porque o cargo de gestor era ocupado pelo presidente ou outro membro do conselho e, em $29 \%$ dos casos, se dava por razões conjuntas.

A separação quase completa de jure existia em apenas $17 \%$ das cooperativas amostradas. Nessas organizações, as condições C1 e C2 eram satisfeitas, porém, C2 era satisfeita apenas parcialmente $^{16}$. A separação quase completa de facto está presente em apenas $4 \%$ do total de cooperativas amostradas.

O estado de São Paulo apresentou a menor frequência de cooperativas que concentram propriedade e decisão de controle, porém, não tinha nenhuma cooperativa com separação quase completa de facto. O Rio Grande do Sul, ao contrário, tinha grande quantidade de cooperativas que não separam os proprietários da decisão de gestão, mas foi o estado que apresentou a maior frequência de cooperativas com separação quase completa de facto.

${ }^{16}$ Em outras palavras, tinha-se que, mas a dedicação do presidente do CA às atividades da cooperativa não era a mínima necessária. Por exemplo, se a dedicação deveria ser de ao menos 43 horas por semana, e se , a dedicação poderia ser próxima de 22 horas semanais.

\section{Conclusões}

A separação entre propriedade e controle é estabelecida para propiciar a desvinculação dos proprietários das decisões de gestão, promover a especialização dos papéis de investidores e gestores e mitigar os problemas de shirking na organização.

O tipo de separação varia entre quase completa, parcial e inexistente e é função de duas condições complementares. A primeira é a delegação dos direitos de controle formal ao conselho de administração, que se manifesta se este órgão possui autoridade formal para nomear ou demitir o agente responsável pela gestão sem consultar a assembleia geral. A segunda condição é a separação das decisões de controle e gestão, de forma que o conselho se responsabilize pelas primeiras enquanto o principal executivo fica com o encargo das segundas. $\mathrm{O}$ índice proposto, composto por duas partes (separação de jure e de facto), permite verificar conjuntamente as duas condições para a observância do grau de separação.

Apenas 38\% das cooperativas da amostra apresentavam separação entre propriedade $\mathrm{e}$ decisão de gestão de jure. Esse resultado foi obtido devido ao elevado número de cooperativas cujo presidente do conselho é eleito diretamente pela assembleia geral e assume a função de agente responsável pela gestão. $\mathrm{O}$ fato de $\mathrm{o}$ presidente do conselho muitas vezes assumir as decisões de controle e gestão implicou elevada incidência $(62 \%)$ de cooperativas cuja estrutura de governança não propicia a separação dos proprietários das decisões de gestão.

A análise conjunta das duas condições para caracterização do grau de separação indicou que $48 \%$ das cooperativas pesquisadas são organizações cuja estrutura de governança concentra a propriedade e as decisões de gestão. As demais apresentam separação parcial (32\%) ou quase completa de jure $(16 \%)$ ou de facto $(4 \%)$. Assim, conclui-se que o Brasil não possui, a exemplo dos países desenvolvidos, um arranjo organizacional predominante; ao contrário, os 
diferentes modelos de governança coexistem no País. Embora grande parcela de cooperativas tenha governança concentrada, há um grupo que já adota modelos de governança que promovem a separação parcial de jure ou de facto entre propriedade e decisão de gestão.

Uma vez que esse trabalho foi desenvolvido com coleta de dados num único momento de tempo, não é possível apontar se existe uma migração de modelos concentrados para quase completos ou vice-versa. Assim, novos estudos devem ser conduzidos para avaliar se os modelos existentes são estruturas definidas ou transitórias e identificar os determinantes da variabilidade de modelos de governança nas cooperativas brasileiras.

\section{Referências bibliográficas}

ALCHIAN, A. A. e DEMSETZ, H. Production, Information Costs, and Economic Organization. The American Economic Review, v. 62, n. 5, p. 777-795, Dec., 1972.

AGHION, P. e TIROLE, J. Formal and Real Authority in Organizations. The Journal of Political Economy, Chicago, v. 105, n. 1, p. 1-29, Feb., 1997.

ARROW, K. J. Control in Large Organizations. Management Science, v. 10, n. 3, p. 397-408, Apr., 1964.

BAKER, G., GIBBONS R. e MURPHY, K. J. Informal Authority in Organizations. The Journal of Law, Economics, \& Organizations, v. 15, n. 1, p. 56-73, 1999.

BAYSINGER, B. e HOSKISSON, R. E. The Composition of Boards of Directors and Strategic Control: Effects on Corporate Strategy. The Academy of Management Review, v. 15, n. 1, p. 72-87, Jan., 1990.

BEBCHUCK, L. A. A rent-protection Theory of Corporate Ownership and Control. Cambridge: Harvard Law School, 1999. p. 47. (Discussion Paper, n. 260).

BERLE, A. A. e MEANS, G. C. The Modern Corporation and Private Property. New York, Macmillan Publishing Co., 1932. p. 396.

BIALOSKORSKI NETO, Sigismundo. Corporate Governance and the Role of the Managers in Brazilian Cooperatives. In: International Center of Research and Information on the Public, Social and Cooperative
Economy, 2003, Oxford England. International Center of Research and Information on the Public, Social and Cooperative Economy. Oxford, England, 2003.

COOK, M. L. The Future of U.S. Agricultural Cooperatives: A Neo-Institutional Approach. American Journal Agricultural Economy, n. 77, p. 1153-1159, 1995.

DEMSETZ, H. e LEHN, K. The Structure of Corporate Ownership: Causes and Consequences. The Journal of Political Economy, v. 93, n. 6, p. 1155-1177, Dec., 1985.

FAMA, E. F. e JENSEN, M. C. Separation of Ownership and Control. Journal of Law and Economics, v. 26, n. 2, p. 301-325, June, 1983.

GINDER, R. G. e DEITER, R. E. Directors and Management. In: COBIA, David (ed.). Cooperatives in Agriculture. New Jersey: Regents/Prentice Hall, 1989. cap. 18, p. $325-338$.

GORTON, G. e SCHMID, F. Corporate governance, ownership dispersion, and efficiency: Empirical evidence from Austrian cooperative banking. Journal of Corporate Finance, n. 5, p. 119-140, 1999.

GRAY, T., W. e BUTTLER, G. Membership Structural Design: A Pilot Test On DHI Cooperatives. Washington, D.C.: USDA, 1994, p. 23. (CS Research Report 131).

HELMBERGER, P. G. Cooperative Enterprise as a Structural Dimension of Farm Markets. Journal of Farm Economics, v. 46, n. 3, p. 603-617, Aug., 1966.

HENDRIKSE, G. W. J. Contingent Control Rights in Agricultural Cooperatives. In: THEURL, T. e MEIJER, E. C. (eds). Strategies for Cooperation. Aachen: Shaker Verlag, 2005, p. 385-394.

HERMALIN, B. E. e WEISBACH, M. S. The Determinants of Board Composition. The RAND Journal of Economics,v. 19, n. 4, p. 589-606, winter, 1988.

HERMALIN, B. E. e WEISBACH, M. S. Board of Directors as an Endogenously Determined Institution: A Survey of the Economic Literature. FRBNY Economic Policy Review, p. 1-27, April, 2003.

HUETH, B. e MARCOUL, P. Incentive Pay for CEOs in Cooperative Firms. American Journal of Agricultural Economics, 91 (5): 1218-1223, 2009.

JENSEN, M. C. e MECKLING, W. H. Theory of the Firm: Managerial Behavior, Agency Costs and Ownership Structure. Journal of Financial Economics, v. 3, n. 4, p. 305360, 1976. 
MIZRUCHI, M. S. Who Controls Whom? An Examination of the Relation Between Management and Boards of Directors in Large American Corporations. The Academy of Management Review, v. 8, n. 3, p. 426-435, Jul., 1983.

OCB, Organização das Cooperativas Brasileiras. Números do Cooperativismo. Dez., 2009. Disponível em: < http:// www.brasilcooperativo.coop.br/site/servicos/biblioteca. asp?CodPastaPai $=40>$. Acesso em dez. 2009.

PELLERVO, CONFEDERATION OF FINNISH COOPERATIVES. Corporate Governance and Management Control in Cooperative. Nov., 2000. Disponível em: <http://www.pellervo.fi/cg/raportti. pdf > Acesso em: 20 de nov. 2009.

RODHES, J. V. Large Agricultural Cooperatives: on the Road to where? Washington, D.C.: USDA, 1987, 16p. Disponível em: <http://www.rurdev.usda.gov/rbs/ pub/sr18/largeag.pdf > Acesso em 15 de junho 2008.

SANTIAGO-CASTRO, M. e BROWN, C. J. Ownership structure and minority rights: A Latin American view. Journal of Economics and Business, n. 59, p. 430-442, 2007.

SERIGATI, F., AZEVEDO, P. F. e ORELLANO, V. I. F. Fidelidade em cooperativas: um estudo empírico com as cooperativas paulistas Anais do XLVII Congresso da Sober, Porto Alegre, 2009.
SHLEIFER, A. e VISHNY, R. W. A survey of Corporate Governance. The Journal of Finance, v. 52, n. 2, p. 737783, Jun., 1997.

SIMON, H. A. The Architecture of Complexity. Proceedings of the American Philosophical Society, v. 106, n. 6, p. 467-482, Dec., 1962.

STAATZ, J. M. The Structural Characteristics of Farmers Cooperatives and their Behavioral Consequences. Washington, D.C.: USDA, 1987, 28p. Disponível em: $<$ http://www.rurdev.usda.gov/rbs/pub/sr18/strcture. pdf $>$ Acesso em 15 de junho 2008.

WAACK, R. S. e MACHADO FILHO, C. P. Administração Estratégica em Cooperativas. In: II Workshop Brasileiro de Gestão de Sistemas Agroalimentares, 1999, Ribeirão Preto. Anais do II Workshop Brasileiro de Gestão de Sistemas Agroalimentares, 1999 .

WILLIAMSON, O. E. Corporate Governance. The Yale Law Journal, v. 93, n.7, p. 1197-1230, Jun., 1984.

WILLIAMSON, O. E. Corporate Boards of Directors: In Principle and in Practice. Journal of Law, Economics, $\mathcal{E}$ Organization, v. 24, n. 2, p. 247-272, Nov., 2007.

ZYLBERSZTAJN, D. Organização de Cooperativas: desafios e tendências. Revista de Administração, v. 29, n. 3, p. 23-32, Jul/Set., 1994. 